\title{
In search of depth
}

\author{
WILLIAM EPSTEIN and TIMOTHY BABLER \\ University of Wisconsin, Madison, Wisconsin
}

\begin{abstract}
Four experiments designed to investigate a visual search task are reported. In each experiment, subjects searched for either a two-dimensional shape rotated in depth among frontal-parallel distractors or a frontal-parallel shape among distractors that were rotated in depth. The principal independent variable was search-set size. In addition, over the four experiments, a variety of spatial arrangements and two axes of rotation in depth were sampled. The chief aim of the experiments was to adduce evidence bearing on the attentional demands of searching for depth. The slopes of the reaction-time (RT) functions were taken as diagnostic. Experiments 1-3 exhibited positive slopes for the RT-set-size function. These slopes appear to be due to a conscious adoption of a serial search strategy by the subjects. When this tendency was suppressed by the procedures of Experiment 4, the slope of the RT-set-size functions did not differ significantly from zero. We conclude that, in agreement with the findings of other studies, slant-in-depth can be detected preattentively.
\end{abstract}

Four experiments are reported in this paper. Each experiment engaged subjects in a visual search task. On each trial of the search task, a set of different two-dimensional (2-D) shapes (not drawings) was presented. On targetpresent trials, the target was either a shape rotated in depth among frontal-parallel distractors or a frontal-parallel shape among distractors that were rotated in depth. On target-absent trials, either all of the shapes in the search set were frontal-parallel and the subject was instructed to search for a shape rotated in depth, or all of the shapes were rotated in depth and the subject was instructed to search for a frontal-parallel shape. In all of the experiments, the size of the search set was varied from trial to trial.

In Experiments 1 and 2, the shapes comprising the search set were arranged with their centers occupying the frontal-parallel plane. In Experiment 1, the shapes were rotated about their central vertical axes. In Experiment 2, the shapes were arranged as in Experiment 1, except that they were rotated about their central horizontal axes. In Experiment 3, each shape comprising the search set occupied a different depth plane. In Experiment 4, a procedure was introduced to discourage the subjects from deliberately adopting a serial search strategy.

The visual search task has been used to assess the attentional demands of perceptual and cognitive operations (e.g., see Duncan, 1985; Egeth, Jonides, \& Wall, 1972; Julesz, 1984; Treisman, 1982; Treisman \& Gelade, 1980; Treisman \& Gormican, 1988; Treisman \& Souther, 1985). The slope of the function relating reaction time (RT) to set size is the diagnostic feature: a slope of zero implicates preattentive operations-that is, operations that are

This research was supported by Research Grant BNS-870658 from the National Science Foundation to William Epstein. Address correspondence to William Epstein, Department of Psychology, University of Wisconsin, Madison, WI 53706. automatic and are executed without allocation of attention; a positive linear slope implicates operations that require allocation of attention in a serial search.

Detection of perturbances of depth is supported by modular processes, such as computation of stereopsis, and is highly practiced. For this reason, detection of perturbances of depth (e.g., detection of a shape rotated in depth among frontal-parallel distractors) seems to be a plausible candidate for designation as a preattentive operation. The results of earlier studies (Epstein \& Babler, 1989; Nakayama \& Silverman, 1986; Ramachadran \& Plummer, 1989) are consistent with this intuition. The present study of visual search was designed to contribute new evidence.

\section{EXPERIMENT 1}

\section{Method}

Subjects. The subjects were 10 introductory psychology students at the University of Wisconsin-Madison.

Apparatus. The apparatus consisted of a two-field tachistoscope, a microprocessor that controlled the tachistoscope fields, and a twobutton response panel. Each field in the tachistoscope had a carousel with eight locations. At each location, a shape mounted on a thin (3-mm diam.) black vertical stalk could be presented. Only a small segment of the stalk was visible; the visible segment of the stalk and its appearance was the same for all shapes in the display. The shapes could be positioned either in the frontal-parallel plane or rotated in depth by $60^{\circ}$ about the vertical axis. The displays were viewed from about $70 \mathrm{~cm}$ and subtended a maximum visual angle of $12.2^{\circ}$.

Stimuli. The subjects viewed five different sets of shapes, with each set consisting of a prototype shape and one complementary shape. The prototype shapes included a circle, a square, and three other shapes chosen from Vanderplas and Garvin's (1959) compilation of irregularly contoured shapes. The complementary shapes were derived from the projection of the prototype when it was rotated in depth by $60^{\circ}$ about the vertical axis with the right edge forward. Multiple replicas of these shapes were constructed from white posterboard and mounted on black stalks. All shapes were approximately $3 \mathrm{~cm}$ in height. 
The displays consisted of a horizontal arrangement of two, three, four, or five shapes. No display had more than one shape from the same prototype set. The shapes were either oriented in the frontalparallel plane or rotated in depth by $60^{\circ}$ about the vertical axis. However, only prototype shapes were presented in a rotated orientation, whereas only complementary shapes were presented in the frontal-parallel plane. Note that a prototype shape rotated $60^{\circ}$ about the vertical axis had the exact same projective shape as its complementary shape presented in the frontal-parallel orientation. Consequently, projective shape alone did not determine orientation.

On target-present trials, only one shape in the display was situated in the designated target orientation. Consequently, on trials that had a rotated shape as the target, one shape in the display was in a rotated position while all other shapes were oriented in the frontal-parallel plane; on trials that had a frontal-parallel shape as a target, one shape was in the frontal-parallel position while all other shapes were rotated in depth. No trials had more than one shape of the designated target orientation. On target-absent trials, no shapes in the display were situated in the designated target orientation.

The following restrictions applied across all set sizes and within each set of 80 trials: (1) rotated targets were as frequent as frontalparallel targets, (2) targets were present on $50 \%$ of the trials, (3) targets appeared equally often in each of the five display locations, and (4) each of the five shapes appeared as a target equally often. Four trials of each set size appeared within each block of 16 trials.

Procedure. The subjects' task was to search each display for a designated orientation. The two possible target orientations (rotated in depth and frontal-parallel) were demonstrated with a sample display prior to the experimental trials. Each trial was initiated by oral designation of the target orientation: the experimenter spoke the words "rotated" or "face on." Approximately $3 \mathrm{sec}$ later, a warning tone sounded, which signaled the presentation of the display $1 \mathrm{sec}$ later. The display remained visible until the subject completed a response. A positive search required a press of a button labeled "yes," whereas a negative search required a press of the "no" button. A short tone sounded following an incorrect response. Trials with incorrect responses were repeated in random order at the end of each block of 16 trials. The subjects were instructed to respond as quickly as possible without sacrificing accuracy. In addition, the subjects were informed that the designated target would be present on $50 \%$ of the trials.

There were two target orientations (rotated, frontal-parallel), two response types (positive, negative), four set sizes $(2,3,4,5)$, and five possible target locations, yielding $\mathbf{8 0}$ different types of trials. These 80 trials were presented in a random order with the restric- tion that 4 trials of each set size appeared within each block of 16 trials. The 80 different trials were presented in a separate random order on four occasions, for a total of 320 experimental trials.

Each subject participated in four 1-h sessions. Each session consisted of 32 practice trials and 80 experimental trials. Practice for each session consisted of a random selection of four different trials for each set size. This block of 16 trials was presented twice in a random order, for a total of 32 practice trials per session.

\section{Results}

Reaction time. Figure 1 plots the mean correct RT across the different conditions. RTs less than $200 \mathrm{msec}$ and greater than $3 \mathrm{sec}$ were discarded.

The overall pattern of results is apparent in Figure 1. The search-time slopes were determined by linear regression. The frontal-parallel target slopes for present and absent responses were quite steep- 86.8 and $123.8 \mathrm{msec}$ per item, respectively. In contrast, the rotated-target slopes were relatively shallow- -37.1 and $25.7 \mathrm{msec}$ per item for target-present and -absent trials, respectively.

An analysis of variance was performed on the RT data. Searches for frontal-parallel targets $(M=1,045 \mathrm{msec})$ resulted in significantly longer $R T s$ than did searches for rotated targets $(M=924 \mathrm{msec})[F(1,9)=15.58, p<$ $.01]$. There was an overall significant effect for set size $[F(3,27)=72.50, p<.001]$, but no significant effect for target presence/absence. In addition, there was a significant interaction of set size $\times$ target type $[F(3,27)=$ $28.53, p<.001]$. A three-way interaction of set size $\times$ target type $\times$ target presence/absence $[F(3,27)=3.40$, $p<.05]$ revealed a different pattern of results for frontalparallel and rotated targets.

A separate analysis of the data from the rotatedtarget trials showed a significant effect of set size $[F(3,27)=12.02, p<.001]$, but no significant effect for target presence/absence and no set size $\times$ target presence/absence interaction for searches of rotated targets.

A separate analysis of frontal-parallel target trials showed a strong effect of set size $[F(3,27)=81.13, p<$ $.001]$. There was no significant effect for target presence/

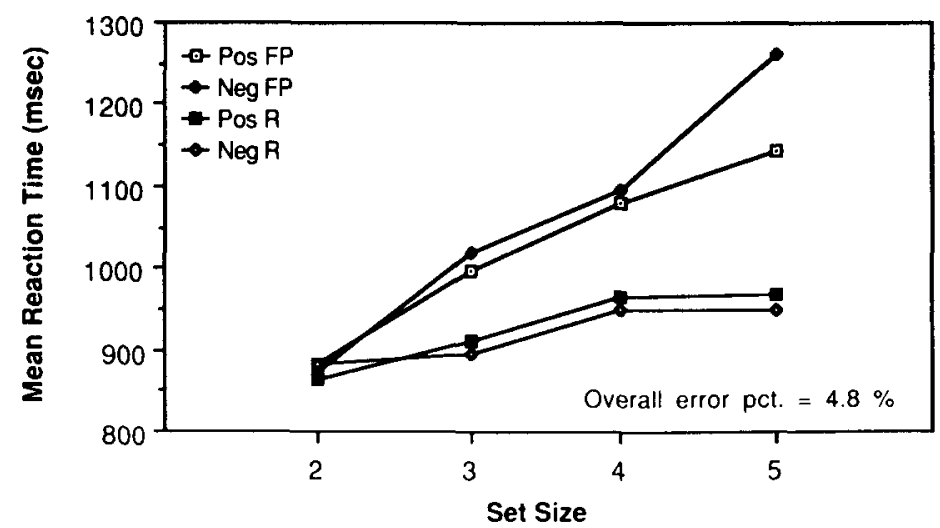

Figure 1. Reaction time as a function of search-set size for the four search conditions in Experiment 1: positive (Pos) and negative (Neg) searches for frontalparallel (FP) and rotated (R) targets. 
absence and no set size $\times$ target presence/absence interaction for frontal-parallel target trials.

Error rates. Error rates averaged $4.8 \%$ across conditions and increased slightly with set size $[F(3,27)=3.40$, $p<.05]$. In addition, searches for frontal-parallel targets resulted in significantly more errors than did rotated-target searches $[F(1,9)=29.24, p<.001]$. Similarly, there were significantly more errors on positive (target-present) trials than on negative (target-absent) trials $[F(1,9)=$ $34.61, p<.001]$. These differences appear to be carried by the large increase in errors for positive frontal-parallel target trials. The mean percentage of errors for positive frontal-parallel trials was 11.4 , compared to an error percentage of only $\mathbf{2 . 6}$ for the remaining conditions.

\section{Discussion}

Contrary to the intuition that motivated this study, the slopes of the RT functions suggest that detection of depth is not preattentive.

The findings of faster RTs for rotated targets and steeper slopes for frontal-parallel targets was unexpected. This asymmetry led us to reconsider the nature of the task for the subjects under the two target conditions. Our original conception construed the task as involving the perceptual encoding of the contents of a set of independent spatial locations (i.e., five spatial locations on trials that presented five shapes). Although this characterization is probably apt when the task called for detection of a frontalparallel target among rotated distractors, it may not be apt when the task called for detection of a rotated target among frontal-parallel distractors. In the latter case, instead of perceptual evaluation of discrete locations, the evaluation may be functionally equivalent to an assessment of a continuous surface for evidence of perturbation. The supposition is that the frontal-parallel distractors in the arrangement used in Experiment 1 form a subjective (perceived) surface owing to the grouping principle of good continuation. The rotated distractors do not conform to the principle of good continuation and hence do not form a surface. Perhaps the difference between the frontal-parallel and rotated-in-depth targets with respect to mean RT and slope is due to this hypothesized difference in the demands of the tasks. Experiment 2 was designed to test this hypothesis.

\section{EXPERIMENT 2}

The hypothesis implies that if the target rotated in depth and the target in the frontal-parallel plane are both presented in an arrangement that fosters surface formation, then searching for the two types of targets should exhibit equivalent mean RTs and slopes. The aim of Experiment 2 was to test this prediction.

The linear spatial arrangement that was used in Experiment 1 was duplicated, with one exception. In Experiment 1 , the shapes were rotated about their vertical axes; in the present experiment, the forms were rotated about their horizontal axes. Rotating a set of forms to the same degree about their horizontal axes was expected to favor generation of a continuous perceptual surface. Under these circumstances, both the frontal-parallel target among rotated distractors and the rotated target among frontalparallel distractors can be distinguished as disruptions of a perceptually continuous surface; hence, both targets should yield comparable mean RTs and comparable shallow slopes.

\section{Method}

Subjects. The subjects were 10 paid volunteers from the University of Wisconsin-Madison community.

Apparatus. The apparatus was identical to that used in Experiment 1.

Stimuli. The subjects viewed five different sets of shapes, with each set consisting of a prototype shape and a complementary shape. The five prototype shapes were identical to those used in Experiment 1 . The complementary shapes were different from those used in Experiment 1; they were derived from the projection of the prototype when it was rotated in depth by $60^{\circ}$ about the horizontal axis with the bottom edge forward. Only prototype shapes were presented in a rotated orientation, whereas only complementary shapes were presented in the frontal-parallel plane.

The displays consisted of a linear, horizontal arrangement of two, three, four, or five shapes. Whereas in Experiment 1, the prototype shapes were rotated by $60^{\circ}$ about the vertical axis, in the present experiment, the shapes were rotated by $60^{\circ}$ about the horizontal axis with the bottom edge forward. The perceptual effect of rotating a set of forms to the same degree about the same horizontal axis is immediately apparent: the rotated forms constitute a continuous perceptual surface. In Experiment 1, only a series of frontalparallel shapes could produce a continuous perceptual surface.

All other aspects of the stimuli were the same as in Experiment 1.

Procedure. The subjects' task was the same as that in Experiment 1 .

\section{Results}

Reaction time. The principal results are shown in Figure 2. As in Experiment 1, searches for frontalparallel shapes $(M=814 \mathrm{msec})$ resulted in longer $\mathrm{RTs}$ than did searches for rotated shapes $(M=758 \mathrm{msec})$ $[F(1,9)=6.41, p<.05]$. The effect of set size was also significant $[F(3,27)=32.10, p<.001]$, with response times increasing with set size.

Negative searches for frontal-parallel and rotated targets yielded similar slope values-34.4 and $28.0 \mathrm{msec}$ per item, respectively. Positive searches, however, produced a steeper increase with set size for frontal-parallel targets than for rotated targets $[F(3,27)=3.57, p<.05]$. Positive searches for frontal-parallel targets yielded a slope of $49.2 \mathrm{msec}$ per set item, whereas positive searches for rotated targets yielded a slope of only $22.1 \mathrm{msec}$ per item.

There was no significant effect of the absence/presence of the target and no significant two-way interactions. The three-way interaction of set size $\times$ target type $\times$ target absence/presence, however, was significant $[F(3,27)=$ $4.74, p<.01]$.

Error rates. Error rates averaged 5.2\% across conditions and did not increase significantly with set size. Posi- 


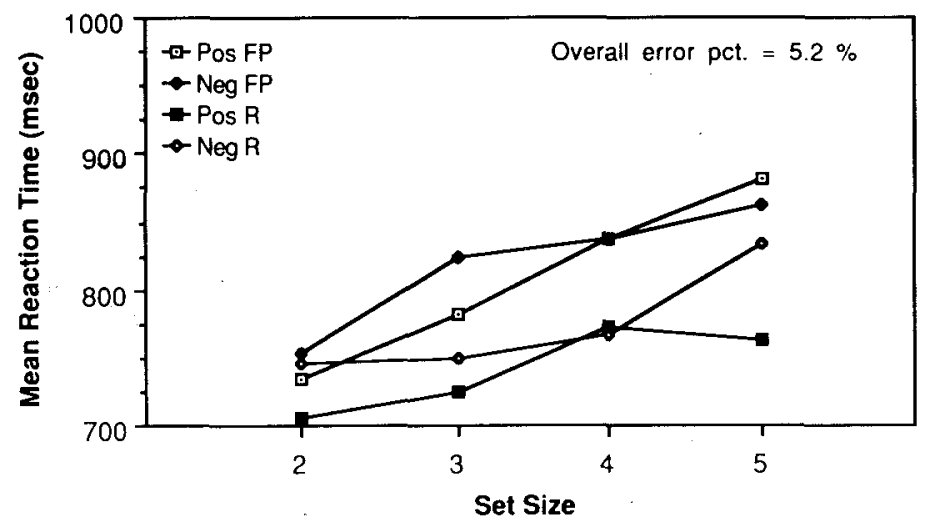

Figure 2. Reaction time as a function of search-set size for the four search conditions in Experiment 2: positive (Pos) and negative (Neg) searches for frontal-parallel (FP) and rotated (R) targets.

tive searches $(M=6.5 \%)$ yielded slightly more errors than did negative searches $(M=3.9 \%)[F(1,9)=13.50$, $p<.01]$. There were no other significant main effects or interactions, except for a significant three-way interaction of set size $\times$ target type $\times$ target absence/presence $[F(3,27)=3.50, p<.05]$.

\section{EXPERIMENT 3}

In Experiments 1 and 2, all of the shapes comprising each search set were located at approximately the same (negligibly different) distance from the subject. This arrangement may have encouraged the subjects to adopt a serial search strategy. Conscious adoption of a serial search strategy may have masked the occurrence of preattentive detection of depth. Experiment 3 was designed to evaluate this possibility by locating each of the shapes at a different distance in a nonlinear arrangement.

\section{Method}

Subjects. The subjects were 10 introductory psychology students at the University of Wisconsin-Madison.

Apparatus. The apparatus was identical to that used in Experiments 1 and 2 .

Stimuli. The shapes used in Experiment 1 served as stimuli. Each shape occupied a different depth plane in one of five positions on the perimeter of an imaginary circle. The diameter of the circle was $11 \cdot \mathrm{cm}$; the distance from the center of the circle to the viewing aperture was $75.5 \mathrm{~cm}$. Figure 3 presents a top view of the display. There was never any interposition of shapes within any of the displays.

Procedure. The subjects' task was the same as in Experiments 1 and 2 .

\section{Results}

Reaction time. Figure 4 shows the mean RTs. An analysis of variance on the RT data revealed a significant increase in search time with set size $[F(3,27)=113.77$, $p<.001]$ and greater search times for trials with absent versus present targets $[F(1,9)=95.05, p<.001]$. The rotated-target slopes for present and absent responses were both quite steep-91.8 and $168.2 \mathrm{msec}$ per item, respectively. The frontal-parallel target slopes were also steep130.8 and 188.0 msec per item for target-present and -absent trials, respectively. The steeper slope for targetabsent trials than for target-present trials is shown by a significant set size $\times$ target absence/presence interaction $[F(3,27)=15.57, p<.001]$.

The overall search times for rotated versus frontalparallel targets did not vary significantly. There was a significant set size $\times$ target type interaction $[F(3,27)=$ $4.80, p<.05$ ], which may reflect slightly steeper slopes for frontal-parallel target trials. However, a separate analysis of the target-present trials showed no such interaction. Therefore, the set-size effect for target-present trials were essentially identical for frontal-parallel and rotated targets. The absence of a three-way set size $\times$ target type $\times$ target absence/presence interaction also suggests a similar pattern of results for frontal-parallel and rotated targets.

Error rates. Error rates averaged 9.2\% across conditions. There was a significant effect of set size $[F(3,27)=$ $6.60, p<.01]$, which was due primarily to a slight elevation in error rates for displays with three shapes. In addition, error rates were slightly higher for target-present trials than for target-absent trials $[F(1,9)=13.33, p<$ $.01]$, and for frontal-parallel targets than for rotated-target trials $[F(1,9)=14.43, p<.01]$.

\section{EXPERIMENT 4}

The results of Experiments 1 and 2 gave no evidence of preattentive detection of slant-in-depth. And the results of Experiment 3 corroborated these findings under different conditions.

Despite the results of Experiment 3, our concern that the findings may be artifacts of a deliberate adoption of a serial search strategy remains. One factor that may have primed serial search in Experiments 1 and 2 is the leftto-right alignment of the members of the search set. This spatial arrangement might act in cooperation with an es- 


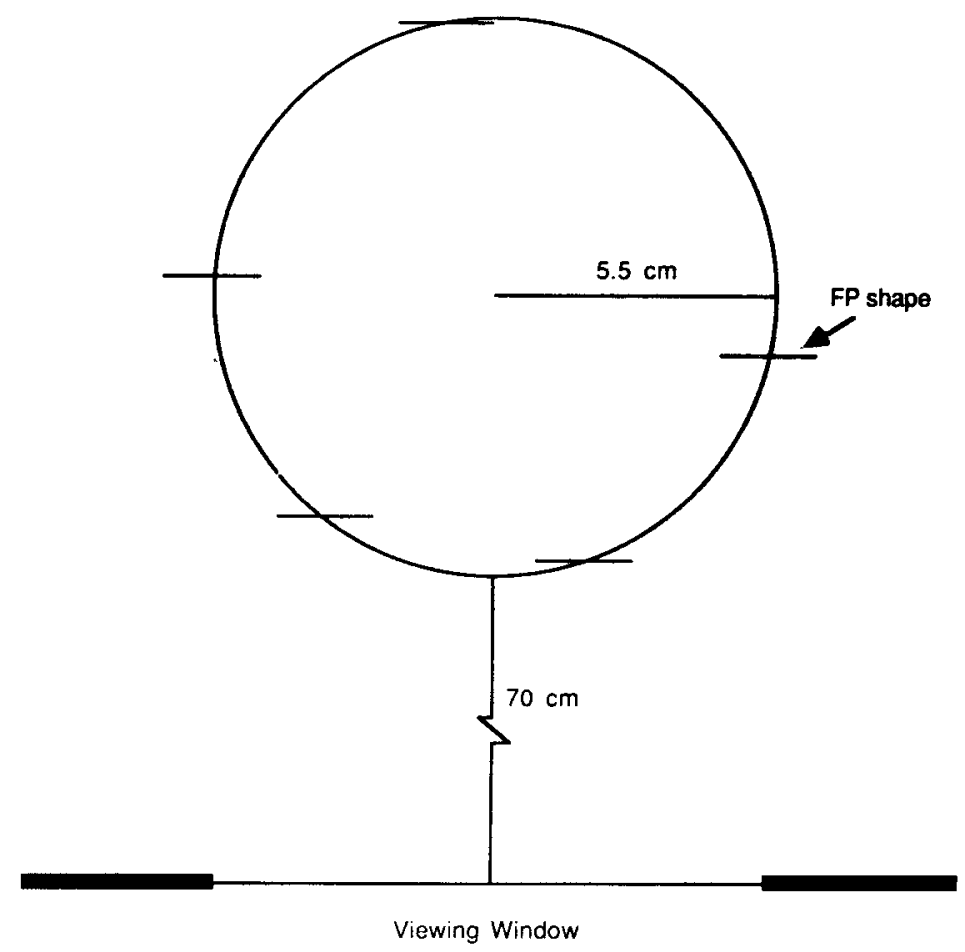

Figure 3. Top view of the spatial arrangement of a sample display in Experiment 3. Each shape occupied a different depth plane in one of five positions on the perimeter of an imaginary circle. The five positions along the imaginary circle were chosen such that no interposition of the shapes would occur in any display. This sample display represents a trial with a negative search for a rotated target for Set Size 5.

tablished preference for a left-to-right search to foster serial search. Locating the shapes in different depth planes, as was done in Experiment 3, retained the leftto-right arrangement of the egocentric locations of the members of the search set.

An explanation is in order. We assume that many discrimination tasks for which it is plausible to postulate an automatic process sufficient for execution of the task may also be executed by a serial search. If, in fact, conditions of the task encourage a serial search, then the outputs of the putative automatic process will be masked. In general, in order to exhibit the products of the automatic operation, possibilities for a serial search must be minimized or serial search must be suppressed. These were the considerations leading to Experiment 4.

The stimuli used in Experiment 4 and their spatial arrangement duplicated the conditions of Experiment 2 in all respects. Experiment 4 was conducted in two stages.

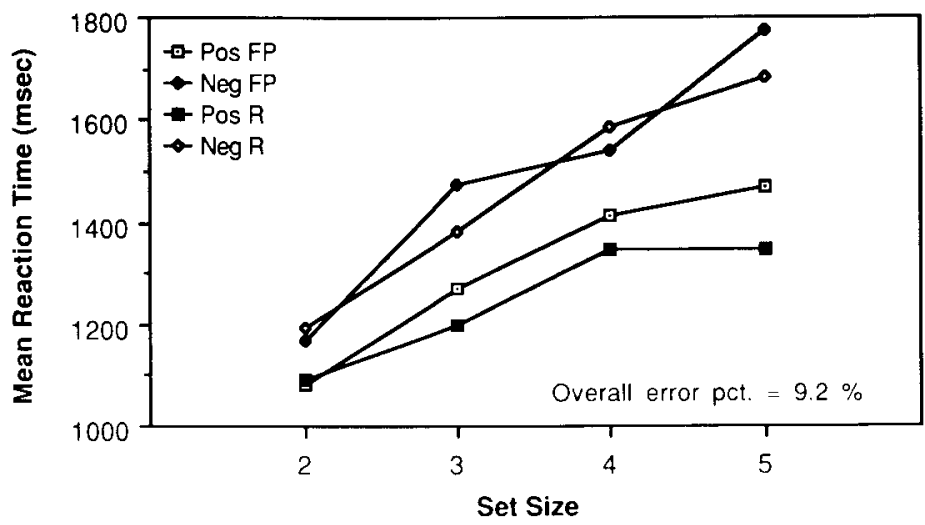

Figure 4. Reaction time as a function of search-set size for the four search conditions in Experiment 3: positive (Pos) and negative (Neg) searches for frontalparallel (FP) and rotated (R) targets. 
The first stage was designed to discourage the deliberate adoption of a serial search strategy. The second stage duplicated the procedure of Experiment 2 in all respects. If the appearance of a serial search in Experiments 1-3 is due to deliberate adoption of a serial search strategy, and if the first stage of Experiment 4 succeeds in suppressing this tendency and such suppression carries over to the second stage, then the results of the replication of Experi- ment 2 should look very different than those of the original experiment: flat or very shallow slopes should appear.

Two steps were taken to discourage the subjects from adopting a serial search strategy during the initial three sessions that comprised the first stage of Experiment 4. First, for all set sizes, the exposure duration was fixed at three standard deviations below the mean RT for the two-item search set in Experiment 2. If the mean RTs in
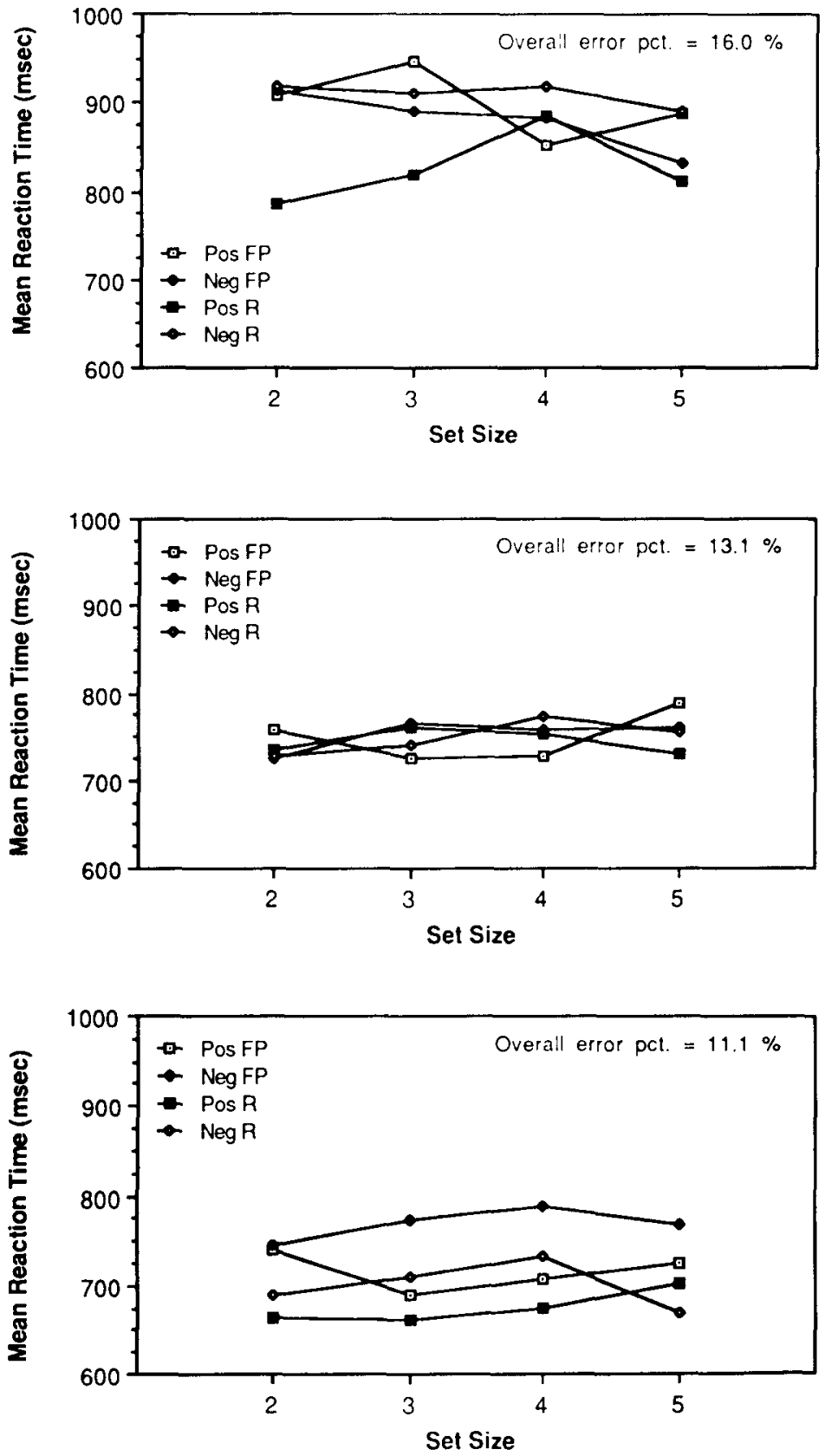

Figure 5. Reaction time as a function of search-set size for the four search conditions in Session 1 (top), Session 2 (middle), and Session 3 (bottom) in Experiment 4: positive (Pos) and negative (Neg) searches for frontal-parallel (FP) and rotated (R) targets. 
Experiment 2 reflect the time required to carry out a serial search, then capping the exposure duration so severely should make serial search an unprofitable strategy. Second, the instructions were modified to encourage the subjects to execute a parallel search (e.g., "do not try to examine each shape one at a time; try to examine them all simultaneously in a single glance"').

During the second stage, which comprised two sessions, the procedure reverted to the one that prevailed in Experiments 1-3; the experimenter-controlled cap on exposure duration was lifted and exposure duration was determined by the subject's response.

\section{Method}

Subjects. The subjects were 8 introductory psychology students from the University of Wisconsin-Madison.

Apparatus. The apparatus was identical to that used in Experiments 1-3.

Stimuli. The stimuli were identical to those employed in Experiment 2.

Procedure. The instructions to the subjects were adapted from the instructions used in Experiment 2, with the following revisions. The subjects were explicitly discouraged from examining the displays one shape at a time. Instead, they were instructed to view the display in a single glance and to base their response on their initial impression. In addition, the exposure duration of the displays was limited to $398 \mathrm{msec}$ during the first three 1-h sessions. In the final two 1-h sessions, display-exposure duration was not limited; in these sessions, the displays were visible up to the moment when the subject made a buttonpress response.

There were two target orientations (rotated, frontal-parallel), two response types (positive, negative), four set sizes $(2,3,4,5)$, and five possible target locations, which yielded 80 different types of trials. These 80 trials were presented twice during each session, for a total of 160 trials. There were five sessions per subject, completed within a 2-week period.

\section{Results}

Limited exposure duration. The mean RTs across the different conditions for Sessions 1-3 are shown in Figure 5. RTs decreased steadily from session to session $[F(2,14)=9.36, p<.01]$. The mean overall response time was $879 \mathrm{msec}$ in Session 1, $749 \mathrm{msec}$ in Session 2, and $715 \mathrm{msec}$ in Session 3. Searches for frontal-parallel targets $(M=794 \mathrm{msec})$ took significantly longer than searches for rotated targets $(M=768 \mathrm{msec})[F(1,7)=$ $7.09, p<.05]$. There was no significant effect of set size on RT across the initial three sessions. In Session 3, the slopes were -2.9 and $13.1 \mathrm{msec}$ per item for positive searches for frontal-parallel and rotated targets, respectively. Negative searches for frontal-parallel and rotated targets yielded slopes of 8.1 and $-4.1 \mathrm{msec}$ per item, respectively.

Error rates appeared to decrease across the initial three sessions, but this change was not statistically significant. Error rates were $16.0 \%$ in Session 1, $13.1 \%$ in Session 2, and $11.1 \%$ in Session 3. Frontal-parallel targets ( $M=16.0 \%$ errors) produced significantly more errors than did rotated targets $(M=11.2 \%)[F(1,7)=$ $6.72, p<.05]$. In addition, positive searches $(M=$ $15.4 \%$ errors) produced more errors than did negative searches $(M=11.8 \%)[F(1,7)=7.88, p<.05]$. There was no significant effect of set size on error rates across the initial three sessions.

Unlimited exposure duration. The data from the final two sessions were pooled for analysis. Figure 6 shows the mean RTs across the different conditions. RT did not vary significantly with set size. Positive searches for frontal-parallel and rotated targets yielded slope values of 3.9 and $6.1 \mathrm{msec}$ per item, respectively. Negative searches produced slopes of -4.1 and $-7.0 \mathrm{msec}$ per item for frontal-parallel and rotated targets, respectively. As in Experiment 2, searches for frontal-parallel targets $(M=695 \mathrm{msec})$ produced slightly longer response times than did searches for rotated targets $(M=628 \mathrm{msec})$ $[F(1,7)=33.35, p<.01]$. In addition, negative search trials $(M=677 \mathrm{msec})$ took longer than positive search trials $(M=646 \mathrm{msec})[F(1,7)=5.68, p<.05]$. There were no other significant main effects or interactions for RT.

Error rates averaged $8.5 \%$ across conditions and did not vary significantly with set size. However, the subjects did make more errors in searching for frontal-parallel tar-

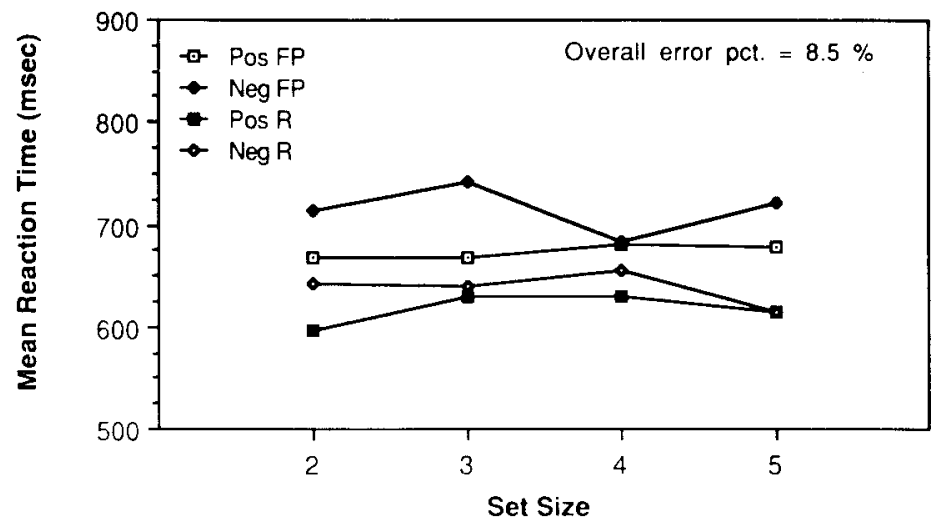

Figure 6. Reaction time as a function of search-set size for the four search conditions in Sessions 4 and 5 (unlimited exposure duration) in Experiment 4: positive (Pos) and negative (Neg) searches for frontal-parallel (FP) and rotated (R) targets. 
gets $(M=10.6 \%)$ than in searching for rotated targets $(M=6.5 \%)[F(1,7)=13.78, p<.01]$. There were no other significant main effects or interactions for error rates.

\section{GENERAL DISCUSSION}

The principal aim of these experiments was to adduce evidence bearing on the attentional demands of detection of depth. In earlier work (Epstein \& Broota, 1986; Epstein \& Lovitts, 1985), a model of processing shape-at-a-slant was promoted in which representation of slant-in-depth was presumed to be preattentive. More recently, we (Epstein \& Babler, 1989) offered the results of two experiments deploying the concurrent task paradigm as evidence that perception of the slant-in-depth of single isolated shapes is automatic. Other evidence bearing on the automaticity of depth detection was provided by Nakayama and Silverman's (1986) demonstration that visual search for a target defined by its position in stereoscopic space (either in the fixation plane or in front) was independent of set size. Also working with stereoscopic displays, Ramachadran and Plummer (1989) reported that a single element that occupied an anomalous depth orientation (e.g., all the lines, or "needles" comprising the stereogram tilted toward the observer except for one, which was tilted in the opposite direction) "popped-out preattentively." The results of the current Experiment 4 add to the earlier body of evidence, in this case for palpable 2-D shapes in 3-D space.

We do not propose to ignore the results of Experiments $1-3$, but we contend that the lesson to be learned from those experiments is concerned more with the boundary conditions for application of the visual search paradigm than with detection of slant-in-depth. A visual search that can be successfully executed on the basis of preattentive outputs will not be irrepressibly executed in this manner. If serial allocation of attention is a plausible alternative procedure, and if the circumstances prime serial search, then a serial search strategy will be adopted. Under these circumstances, the output of the preattentive process will not be detected. ${ }^{1}$

The curious effects of target type merits comment. In Experiments 1, 2, and 4, rotated targets were detected faster than frontal-parallel targets. In Experiment 3, which presented each shape in a different depth plane, RTs for rotated and frontal-parallel targets did not differ.

These effects might appear to be a degenerate case of the type of search asymmetry discovered by Treisman and her colleagues (Treisman \& Gormican, 1988; Treisman \& Souther, 1985), who reported that detection is faster when preattentive features are present in the target and absent in the distractors than when the target is distinguished by the absence of a particular preattentive feature that is exhibited by the distractors. In Experiments 1, 2 , and 4 of the current study, if a positive value for binocular disparity associated with the rotated target is construed as a preattentive feature and the zero disparity associated with frontal-parallel distractors is taken as an instance of absence of the feature, then the difference between the mean RTs for the two types of targets might be an example of the class of search asymmetries described by Treisman and her colleagues. In Experiment 3, the allocation of the shapes to different depth planes and the attendant disparities associated with all of the shapes would overwrite the putative effects of the rotation of individual shapes. An objection to this account springs to mind: The account assigns special status (conspicuity) to positive disparity although there is no warrant for such assignment. Disparity is a continuous variable and so is orientation in depth.

Throughout the foregoing discussion and in our original framing of the question, we have proceeded as if detection of depth was determined by the pickup of a single informational variable. Of course, this supposition is manifestly incorrect. Detection of depth is supported by a diverse set of informational variables (e.g., binocular disparity, shading, optical motion, perspective). And it is not implausible to suppose that although detection of depth supported by one or more of these informational variables may be automatic, detection of depth supported by other variables may require attention. For example, depth detection from stereopsis may be automatic, whereas depth detection from shading may be attentional. Were this hypothetical example to be fact, it would be expected that visual search for depth in displays in which binocular disparity is salient would exhibit characteristics that differ from the characteristics exhibited by search for depth in displays in which shading is salient.

A shortcoming of the type of displays used in our experiments is the absence of control over the informational variables for depth. This shortcoming is obvious when our displays are compared with the stereoscopic displays studied by Nakayama and Silverman (1986) and Ramachadran and Plummer (1989). In these latter cases, the only information for depth was stereopsis. In effect, the question that was addressed in those studies concerns the detection of disparity depth. In our experimental settings, several informational variables were available, including binocular disparity and shading, and we are unable to say whether one or the other of these variables governed detection of depth or whether, in fact, all available variables contributed in a cooperative process.

\section{REFERENCES}

DUnCan, J. (1985). Visual search and visual attention. In M. I. Posner \& O. S. M. Martin (Eds.), Attention and performance (Vol. 11, pp. 85-106). Hillsdale, NJ: Erlbaum.

EGETH, H., Jonides, J., W WLL, S. (1972). Parallel processing of multielement displays. Cognitive Psychology, 3, 674-698.

Epstein, W., \& BAbler, T. (1989). Perception of slant-in-depth is automatic. Perception \& Psychophysics, 45, 31-33.

EPSTEIN, W., BRoOtA, K. D. (1986). Automatic and attentional components in perception of size-at-a-distance. Perception \& Psychophysics, 40, 256-262. 
EPSTEIN, W., \& LovitTs, B. E. (1985). Automatic and attentional components in perception of shape-at-a-slant. Journal of Experimental Psychology: Human Perception \& Performance, 11, 355-366.

Julesz, B. (1984). Toward an axiomatic theory of preattentive vision. In G. Edelman, M. Cowan, \& M. D. Gall (Eds.). Dynamic aspects of neocortical function (pp. 585-612). New York: Wiley.

NaKaYAma, K., \& Silverman, G. H. (1986). Serial and parallel processing of visual feature conjunctions. Nature, 320, 264-265.

Ramachadran, V. S., Plummer, D. J. (1989). Preattentive perception of 3D versus 2D image features. Investigative Ophthalmology \& Visual Science (Suppl. 30), 160.

Treisman, A. (1982). Perceptual grouping and attention in visual search for features and for objects. Journal of Experimental Psychology: $\mathrm{Hu}$ man Perception \& Performance, 8, 194-214.

Treisman, A., \& Gelade, G. (1980). A feature integration theory of attention. Cognitive Psychology, 12, 97-136.

Treisman, A., \& Gormican, S. (1988). Feature analysis in early vision: Evidence from search asymmetries. Psychological Review, 95 , 15-48.

Treisman, A., \& Souther, A. (1985). Search asymmetry: A diagnostic for preattentive processing of separable features. Journal of Experimental Psychology: General, 114, 285-310.

VANDERPLAS, J., \& Garvin, E. A. (1959). The association value of nonsense shapes. Journal of Experimental Psychology, 57, 147-154.

\section{NOTES}

1. In addition to the spatial arrangement, another factor that might have led to the adoption of a serial search strategy is a tendency by subjects to reinterpret the demands of the task as requiring identification of shape-at-a-slant. The instructions to the subjects emphasized that orientation was the target and that identification of shape was neither required nor diagnostic, inasmuch as all shapes would occupy both orientations in depth equally often. Nevertheless, the inclination to process the display to the point of resolving shape-at-a-slant may have been irresistible. According to the model we (Epstein \& Babler, 1989; Epstein \& Lovitts, 1985) have advanced, computation of shape-at-a-slant-that is, generation of an object-centered representation of shape-does require allocation of attention. When more than one shape is present, the computation of object-centered representations will necessarily be serial. Thus, if the subjects in Experiments 1-3 elected to process the contents of the search set fully, the putative automatic detection of depth would be masked. Capping the exposure duration in Experiment 4 would discourage full processing.

(Manuscript received August 7, 1989; revision accepted for publication January 26, 1990.) 